\title{
Treatment of choroid metastasis from lung adenocarcinoma with bevacizumab-containing chemotherapy: A case report
}

\author{
KENICHI MAKABE ${ }^{1}$, KOICHI KURISHIMA ${ }^{2}$, TOSHIHIRO SHIOZAWA ${ }^{2}$, KUNIHIKO MIYAZAKI ${ }^{2}$, \\ GEN OHARA $^{2}$, KATSUNORI KAGOHASHI ${ }^{2}$, HIROAKI SATOH ${ }^{2}$ and NOBUYUKI HIZAWA ${ }^{3}$
}

\author{
Divisions of ${ }^{1}$ Ophthalmology and ${ }^{2}$ Respiratory Medicine, Mito Medical Center, University of Tsukuba, Mito, Ibaraki 310-0015; \\ ${ }^{3}$ Division of Respiratory Medicine, Faculty of Medicine, University of Tsukuba, Tsukuba, Ibaraki 305-8575, Japan
}

Received June 5, 2014; Accepted February 3, 2015

DOI: $10.3892 /$ etm.2015.2893

\begin{abstract}
The occurrence of ocular metastasis from lung cancer is uncommon. The present study reports the case of a 69-year-old female patient with lung adenocarcinoma who was found to have a metastatic lesion in the left choroid at the time of presentation. As the patient was found to have a mutation in the epidermal growth factor receptor, treatment with gefitinib was administered; however, the response was evaluated as a progressive disease. Thereafter, the patient received chemotherapy with carboplatin, pemetrexed and bevacizumab. Radiological imaging revealed shrinkage of the primary lesion and choroidal metastasis, and the visual power of the left eye was also shown to improve. Therefore, the present case report demonstrated the efficacy and safety of systemic bevacizumab therapy in combination with a platinum doublet for the treatment of choroid metastasis, with morphological and functional improvements observed with regard to the choroidal metastatic tumor.
\end{abstract}

\section{Introduction}

Although rare, metastatic choroid tumors are the most common type of intraocular malignancy. The frequency of intraocular metastasis in all patients dying of cancer was reported to be approximately $12 \%$ (1). Lung and breast cancers are two of the predominant tumors to metastasize to the eye $(2,3)$. Among the ocular metastases, the choroid is the most commonly affected site (2). The prognosis for patients with ocular metastases remains inadequate. For these patients life expectancy is reported to be between 2-48 months (median 6-9 months) $(4,5)$. Radiotherapy remains the main type of therapy and allows the majority of patients to maintain a good vision for as long as they are alive (1). Recently, the development of molecular

Correspondence to: Professor Hiroaki Satoh, Division of Respiratory Medicine, Mito Medical Center, University of Tsukuba, Miya-machi 3-2-7, Mito, Ibaraki 310-0015, Japan

E-mail: hirosato@md.tsukuba.ac.jp

Key words: choroid metastasis, lung cancer, bevacizumab targeted therapies, such as humanized antivascular endothelial growth factor, bevacizumab and epidermal growth factor receptor (EGFR)-tyrosine kinase inhibitors, has improved the survival rate of patients with advanced non-small cell lung cancer (NSCLC), without substantially increasing the toxicity (6). The present study reports the case of a patient with NSCLC and choroid metastasis who was successfully treated with bevacizumab-containing chemotherapy, following treatment failure with gefitinib.

\section{Case report}

A 69-year-old female that had been newly diagnosed with a metastatic adenocarcinoma of the lung presented with a one-month history of blurred vision in the left eye. The best corrected visual activity (BCVA) in the left eye was 20/200. A chest radiograph and computed tomography $(\mathrm{CT})$ scan revealed a pulmonary mass in the left upper lobe with ipsilateral mediastinal lymph node swelling (Fig. 1). In addition, pleural fluid was observed in the left lung, while metastases were identified in both lungs, the paraaortic lymph nodes of the abdomen and the right adrenal gland. A brain CT scan revealed a choroidal tumor in the left eye that measured $10.4 \times 6.4 \times 2.6 \mathrm{~mm}$ in size (Fig. 2). Pathological diagnosis of the lung mass, which was obtained via a transbronchial biopsy, revealed an adenocarcinoma. Using the specimen obtained from the primary lesion by bronchoscopy, an EGFR mutation was evaluated using a PCR clamp method. The PCR clamp method preferentially amplifies mutation sequences and detects mutations. The method requires a peptide nucleic acid (PNA) clamp primer and locked nucleic acid (LNA) probe. PNA clamp primers bind to the wild type sequence and suppress their amplification, whereas LNA probes are designed to specifically detect mutant sequences and enhance their amplification in the presence of wild type sequences, since PNA clamp primers competitively inhibit the ability of mutant LNA probes to bind to wild type sequences. To detect mutations of exons 18, 19, 20 and 21, a total of 12 probes were used. The PCR clamp method was performed by Mitsubishi Kagaku Bio-Clinical Laboratories, Inc. (Tokyo, Japan). As the patient had a mutation in the EGFR gene (exon 21 L858R), treatment with gefitinib was initiated. However, the BCVA of the left eye continued to deteriorate to 'mortus manus' (finger movement) and the 
pleural fluid in the left lung increased. Therefore, the response was evaluated as a progressive disease. Subsequently, the patient received chemotherapy with carboplatin (AUC 5, day 1 , q28), pemetrexed $\left(500 \mathrm{mg} / \mathrm{m}^{2}\right.$, day $\left.1, \mathrm{q} 28\right)$ and bevacizumab (15 mg/mg, day 1, q28). A brain CT scan performed after two cycles of the chemotherapy revealed the disappearance of the choroidal lesion (Fig. 3). In addition, an improvement in vision of up to 20/200 was observed. Subsequently, the patient completed four cycles of the chemotherapy and attained a partial response. The patient received additional maintenance chemotherapy with pemetrexed $\left(500 \mathrm{mg} / \mathrm{m}^{2}\right.$, day $\left.1, \mathrm{q} 28\right)$ and bevacizumab (15 mg/mg, day 1, q28). The patient survived for 16 months after the diagnosis of lung adenocarcinoma, with no evidence of deterioration of vision. Written informed patient consent was obtained from the patient's family.

\section{Discussion}

Breast and lung cancers are the most common primary tumors that metastasize to the eye and orbit $(2,3,7)$, and the most commonly affected part of the eye is the vascular uveal tract (7). Within the uvea, the choroid is the most frequently affected site of metastasis (2). As observed in the patient in the present study, the majority of patients have metastatic lesions elsewhere at the time of diagnosis of ocular involvement (8). As a result of such metastasis, the treatment for ocular metastasis may be palliative and the aims for treatment are to maximize the quality of life and restore or preserve vision.

Certain case reports have demonstrated that chemotherapy is efficacious for choroid metastases from NSCLC $(9,10)$. To the best of our knowledge, there have been seven case reports that precisely describe the outcome of bevacizumab-containing chemotherapy for the treatment of choroid metastasis (11-16). The characteristics of these studies are outlined in Table I. In four cases, the patient was diagnosed with adenocarcinoma. However, no information was provided with regard to EGFR gene mutations in any of the patients. In all the cases, improvement was observed in the choroid metastasis, as well as functional improvement in visual power. In four cases, including the present study, systemic bevacizumab-containing chemotherapy was applied (one patient received cisplatin-based therapy and three patients received carboplatin-based chemotherapy) $(11,14,16)$. Kim et al reported successful treatment with oral erlotinib and intravitreal therapy with bevacizumab (12). In the present case, gefitinib was not effective for the intrathoracic and intraocular lesions, despite the detection of a mutation in the EGFR gene. Lai et al reported the successful treatment of a case with intravitreal bevacizumab therapy, without administration of systemic chemotherapy (15). Visual power in this patient was shown to improve; however, the patient succumbed four months after the diagnosis (15). By contrast, D'Antonio et al presented a case who was successfully treated with systemic bevacizumab-containing chemotherapy and survived for over 20 months (14). In the present study, the patient survived for 16 months following the diagnosis of lung cancer. However, the prognosis of choroid metastasis from lung cancer is very poor, with a mean life expectancy of 1.9 months (4). This short survival time is not due to the choroid metastasis itself, but the condition of disease dissemination with the involvement

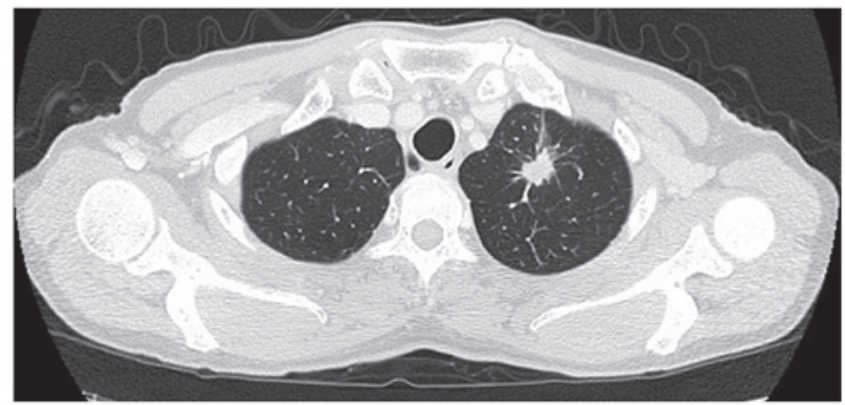

Figure 1. Chest computed tomography scan at the initial presentation revealed a mass in the upper lobe of the left lung with pleural fluid.

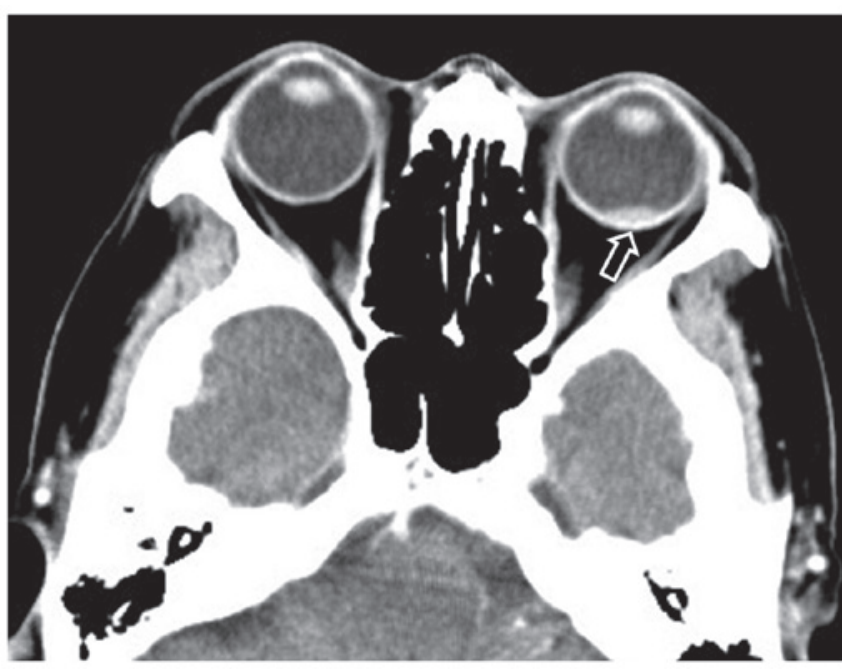

Figure 2. Brain computed tomography scan at the initial presentation revealed a metastatic tumor (size, $10.4 \times 6.4 \times 2.6 \mathrm{~mm}$ ) in the choroid of the left eye (arrow).

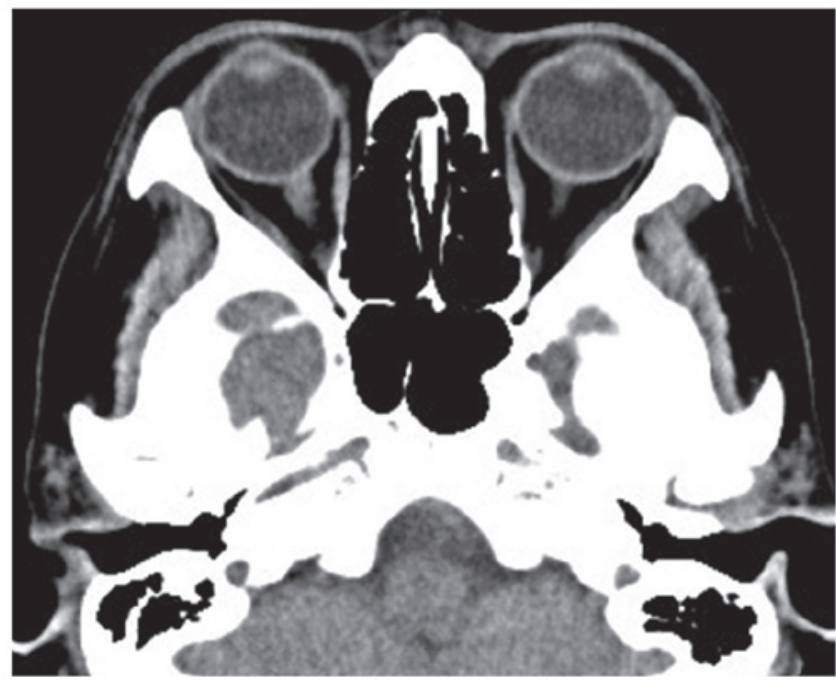

Figure 3. Brain computed tomography scan following two cycles of bevacizumab-containing chemotherapy revealed the disappearance of the choroid tumor in the left eye.

of other vital organs. The four patients treated with bevacizumab and the current patient had metastatic sites other than in the choroid. Singh et al reported two cases treated 


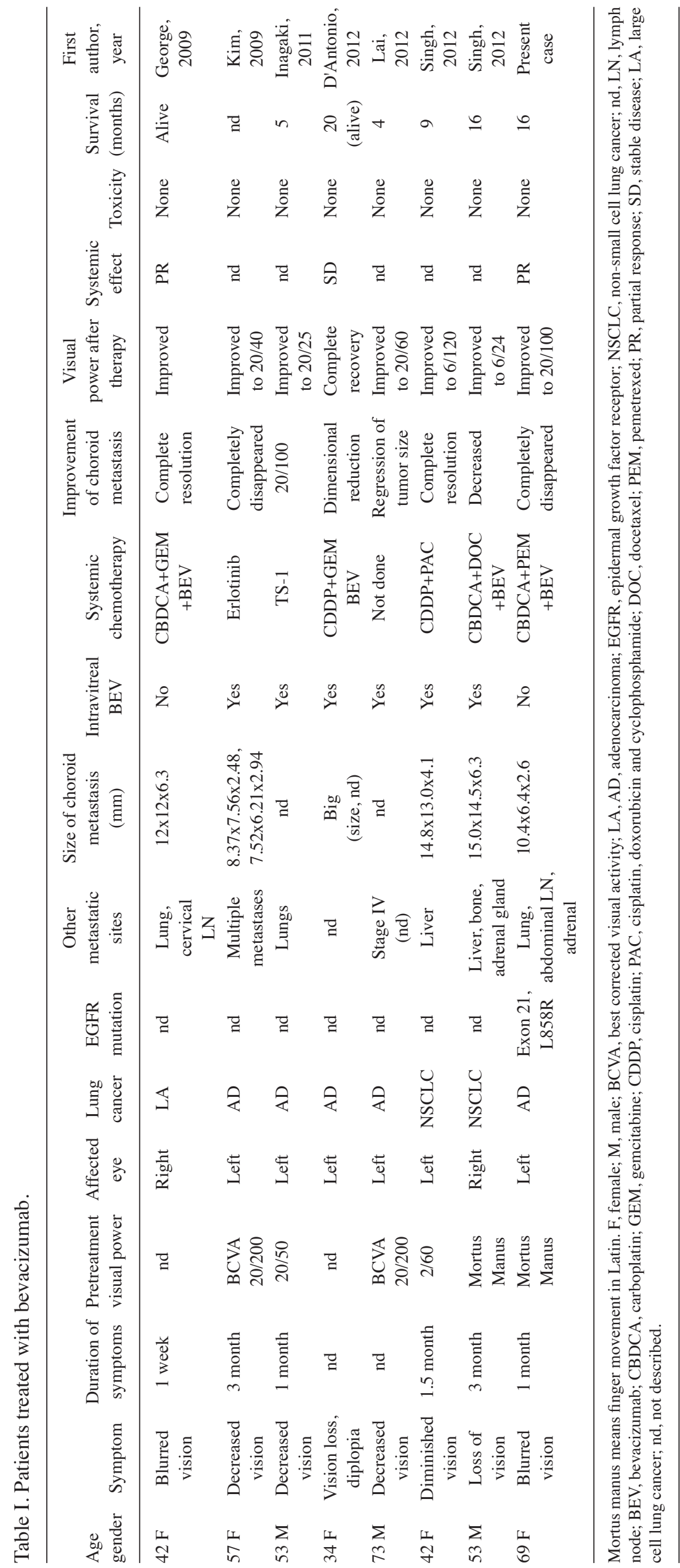


with bevacizumab-containing chemotherapy. In one of these patients, first line chemotherapy without bevacizumab was not effective for the choroid metastasis; however, second line chemotherapy with bevacizumab-containing chemotherapy achieved resolution of the choroid metastasis and visual improvement (16). Notably, the patient in the present study received bevacizumab-containing chemotherapy as the second line treatment and also achieved resolution of the choroid metastasis and visual improvement. These results suggested that systemic chemotherapy with bevacizumab may have an significant role in improving visual power as well as survival of the patients.

Intravitreal bevacizumab administration has been used to treat choroid metastases from organs other than the lungs $(17,18)$. In these two studies, antiangiogenic and antipermeability effects of bevacizumab were observed on the new tumor vessels by fluorescein angiography. These observations demonstrate the obligate and vital dependence of choroidal tumors on angiogenesis, which forms the rationale for the use of intravitreal bevacizumab administration, regardless of the fact that a platinum doublet in combination with bevacizumab is one of the chemotherapy options for the treatment of lung cancer patients with choroid metastasis. Two of the patients who were treated with systemic bevacizumab-containing chemotherapy received intraviteral administration of bevacizumab $(14,16)$ (Table I).

In conclusion, the present case report demonstrated the efficacy and safety of systemic bevacizumab therapy in combination with a platinum doublet for the treatment of choroid metastasis, with resulting morphological and functional improvements in the choroidal metastatic tumor. Therefore, systemic administration of bevacizumab with intravitreal injections may be selected as a therapeutic approach due to the greater potential to produce effective chemotherapeutic concentrations around the metastasis site via the rich choroidal blood supply, which is within the systemic circulation and not protected by the blood-retina barrier.

\section{References}

1. Small W Jr: Management of ocular metastasis. Cancer Control 5: 326-332, 1998

2. Shields CL, Shields JA, Gross NE, Schwartz GP and Lally SE: Survey of 520 eyes with uveal metastases. Ophthalmology 104: 1265-1276, 1997.
3. Soysal HG: Metastatic tumors of the uvea in 38 eyes. Can $\mathbf{J}$ Ophthalmol 42: 832-835, 2007.

4. Kreusel KM, Wiegel T, Stange M, Bornfeld N, Hinkelbein W and Foerster MH: Choroidal metastasis in disseminated lung cancer: frequency and risk factors. Am J Ophthalmol 134: 445-447, 2002.

5. Tsina EK, Lane AM, Zacks DN, Munzenrider JE, Collier JM and Gragoudas ES: Treatment of metastatic tumors of the choroid with proton beam irradiation. Ophthalmology 112: 337-343, 2005.

6. Bunn PA Jr and Thatcher N: Systemic treatment for advanced (stage IIIb/IV) non-small cell lung cancer: more treatment options; more things to consider. Conclusion. Oncologist 13 (Suppl 1): 37-46, 2008.

7. Freedman MI and Folk JC: Metastatic tumors to the eye and orbit. Patient survival and clinical characteristics. Arch Ophthalmol 105: 1215-1219, 1987.

8. Kanthan GL, Jayamohan J, Yip D and Conway RM: Management of metastatic carcinoma of the uveal tract: an evidence-based analysis. Clin Experiment Ophthalmol 35: 553-565, 2007.

9. Battikh MH, Ben Yahia S, Ben Sayah MM, et al: Choroid metastases revealing pulmonary adenocarcinoma resolved with chemotherapy. Rev Pneumol Clin 60: 353-356, 2004 (In French).

10. Shields JA, Perez N, Shields CL, Foxman S and Foxman B: Simultaneous choroidal and brain metastasis as initial manifestations of lung cancer. Ophthalmic Surg Lasers 33: 323-325, 2002.

11. George B, Wirostko WJ, Connor TB and Choong NW: Complete and durable response of choroid metastasis from non-small cell lung cancer with systemic bevacizumab and chemotherapy. J Thorac Oncol 4: 661-662, 2009.

12. Kim SW, Kim MJ, Huh K and Oh J: Complete regression of choroidal metastasis secondary to non-small-cell lung cancer with intravitreal bevacizumab and oral erlotinib combination therapy. Ophthalmologica 223: 411-413, 2009.

13. Inagaki E, Shinoda H, Uchida A, et al: Effect of intravitreal injection of bevacizumab for exudative retinal detachment secondary to metastatic choroidal tumor: Case report. Atarashii Ganka 28: 587-592, 2011 (In Japanese).

14. D'Antonio C, Viterbo A, Romiti A, Enrici MM, Lauro S and Marchetti P: Complete regression of a non-small cell lung cancer choroidal metastasis with intravitreal bevacizumab. J Thorac Oncol 7: 468-469, 2012.

15. Lai CL, Fan KS, Lee YH, Chen HC and Fan WH: Intravitreal administration of bevacizumab in the treatment of choroidal metastasis in a patient with erlotinib-failed pulmonary adenocarcinoma. Lung Cancer 76: 496-498, 2012.

16. Singh N, Kulkarni P, Aggarwal AN, et al: Choroidal metastasis as a presenting manifestation of lung cancer. A report of 3 cases and systematic review of the literature. Medicine (Baltimore) 91: 179-194, 2012.

17. Amselem L, Cervera E, Díaz-Llopis M, et al: Intravitreal bevacizumab (Avastin) for choroidal metastasis secondary to breast carcinoma: Short-term follow-up. Eye (Lond) 21: 566-567, 2007.

18. Kuo IC, Haller JA, Maffrand R, Sambuelli RH and Reviglio VE: Regression of a subfoveal choroidal metastasis of colorectal carcinoma after intravitreous bevacizumab treatment. Arch Ophthalmol 126: 1311-1313, 2008. 